\title{
Study on solar tracking trajectory calculation method with Lagrange interpolation
}

\author{
Zhenguo Zhang ${ }^{1, \text { a }}$, Hongwei Shi ${ }^{1, \mathrm{~b}}$, Yan $\mathrm{Li}^{1, \mathrm{c}}$, Bo Dong ${ }^{1, \mathrm{~d}}$ and Yuanchun $\mathrm{Li}^{1, \mathrm{e}^{*}}$ \\ ${ }^{1}$ Department of Control Engineering, Changchun University of Technology, Changchun 130012, \\ China \\ a15568813658@163.com, bqitiand@163.com, '9y6655@126.com, \\ dongbo@ccut.edu.cn,
}

\begin{abstract}
Keywords: Solar tracking, Lagrange interpolation, Trajectory calculation, Piecewise interpolation. Abstract: This paper presents a novel solar tracking trajectory calculation method, and addresses the problems of low efficiency, computing complex issues of solar tracking control system. Based on the Lagrange interpolation method, the solar trajectory is calculated by using the location information in certain times (day, month and year), and then the sun can be accurately tracked with the method of photoelectric tracking. Finally, simulations are preformed with MATLAB to verify the validity of the proposed method.
\end{abstract}

\section{Introduction}

Solar energy utilization system is exposed wildly attention in modern city life. In some conventional methods, the energy is converted into other forms of energy, and used them. However, in these systems, the solar energies are proportional to the sun's rays on the smooth projection under the same intensity. In order to obtain the vertical gain maximum energy, the real-time accurate tracking to the direction of the sun's rays for solar energy utilization efficiency is very important, and it is the key technology of the solar tracking system.

Scholars have conducted extensive research to it ,which the solar tracking strategy and the choice of system control unit, the literature $[1,2]$ finish the solar position calculation and other control functions use micro controller. On the premise of guarantee accuracy reduces the cost of development, the literature [3] achieve roughly solar track with micro controller as the controller. At present lots of solar automatic tracking control system using visual trajectory tracking combine with photoelectric sensor tracking strategy, the literature $[4,5]$ use photosensitive resistance sensor combined with solar trajectory tracking control strategy, the literature [6] use the silicon photocell sensor combined with solar trajectory tracking control strategy, the literature [7] use the panoramic image processing methods combined with solar trajectory tracking control strategy. It's a very classical control strategy. Solar trajectory tracking control for main role in this system is based on time and location information by calculating a rough location of the sun position, it's tracking error in the range of photoelectric sensors, in order to photoelectric sensor for precise adjustment. If not use the solar trajectory tracking, may cause the sun light outside of the photoelectric sensor detection range, leading to the solar tracking device was unable to complete tracking, so the solar position calculation for the whole tracking system is very important. The literature [8] is proposed based on T-S model to realize the solar position of simplified calculation, the literature [9] by comparing a variety of the solar position calculating model, considering the other influence factors of selecting the optimal model, the literature [10] by simplifying the formula for calculating the solar position to make the solar trajectory algorithm becomes simple. On the solar trajectory tracking in the role of the system, it's very inconvenient in practical application because it's calculation formula is complex, and the influence on the speed of system operation.

In this paper, an interpolation method is used to calculate the solar location, it can significantly reduce the computation on the premise of guarantee accuracy. Store up part of the solar location data using micro controller, respectively calculate the solar trajectory of the solar elevation angle and the solar azimuth angle using interpolation algorithm. As control amount respectively control the solar 
elevation angle and the solar azimuth angle actuator to realize the sun tracking. It can improve the efficiency of the system, simplify the programming and development difficulty on the premise of meeting the requirements of the function and precision.

\section{Problem Statement}

The solar position calculation used more by the area of the time the solar declination angle, solar hour angle, and variable, using the spherical triangle formula or vector method to calculate the horizontal coordinate of the position of the solar elevation angle and azimuth angle as shown in Fig. 1, the solar is depending on the declination angle and the center of the earth wire and the angle between the equatorial plane, the earth is also called the declination angle of the sun; Angle when the sun is the center of the plane angle, namely from the celestial meridian spot along the celestial equator measured distance to the sun in the angle of the circle.

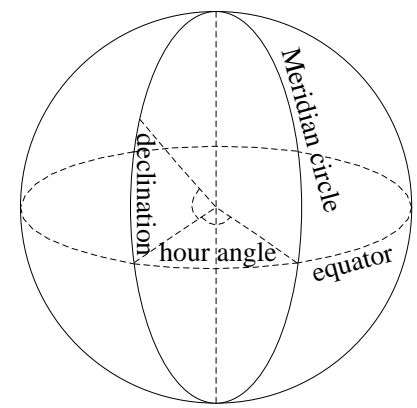

Fig. 1 Solar declination angle and solar hour angle

Because of the uncertainty of the motion orbit the sun, stars perturbation factors such as interference in the solar position accurate calculation is very complicated, some scholars put forward some approximation method for computation of the solar position, Spencer method considering precision and computation usually as a day of apparent trajectory tracking solar position algorithm is the best choice, but still need a lot of trigonometric function is calculated. And practical application, due to the complexity of the calculation and calculation, accurate get exact timing and location information can't wait for a reason, lead to direct calculation results cannot be used to directly control the sun tracking device to track, and need to use a combination of the photoelectric tracking, real-time measuring the sun position, it can realize accurate tracking the sun. But in the implementation of control, there will be a solar direction deflection caused by the photoelectric sensor can't detect the sun's rays (for example, this morning when the solar rises just or cloudy sunny days afterwards) .Need to see at this time, trajectory tracking control drive the sun tracking device within a certain range alignment, after adopting photoelectric tracking device for accurate alignment. Under this kind of functional requirement, inspect, trajectory tracking of the sun's position does not require precise tracking, so you can use the lower precision, effectively resist known-plaintext attack but the approach to the solar position is calculated. Based on this requirement, this paper proposes a Spencer algorithm based on the formation of point data, in the practical application of the interpolation method to calculate the sun position. The formula for calculating the solar position is as follows:

$$
\begin{gathered}
\sin \alpha=\sin \varphi \sin \delta+\cos \varphi \cos \delta \cos \omega \\
\sin \beta=\frac{\cos \delta \sin \omega}{\cos \alpha} \\
\cos \beta=\frac{\sin \alpha \sin \beta-\sin \delta}{\cos \alpha \cos \beta}
\end{gathered}
$$

In equation (1): $\alpha \longrightarrow$ Solar elevation angle;

In equation (2): $\beta-$ Solar azimuth angle;

In equation (1): $\delta \longrightarrow$ Solar declination angle; 
In equation (1): $\omega-$ Solar hour angle;

In equation (1): $\varphi-$ Latitude.

while $\delta$ and $\omega$ are all functions of time.

From the above equation, when the observation site is determined, $\varphi$ is constant, so the mathematical model of the solar position can be expressed as:

$$
\left\{\begin{array}{l}
\alpha=f_{1}(\delta(t), \omega(t)) \\
\beta=f_{2}(\delta(t), \omega(t))
\end{array}, 0<t<24\right.
$$

In the equation (4): $f_{1}(\delta(t), \omega(t))$ express a function relationship between $t$ and $\alpha$.

In the equation (4): $f_{2}(\delta(t), \omega(t))$ express a function relationship between $t$ and $\beta$.

It can be seen that the solar motion can be simplified to two simple motions of the solar zenith angle, the time varying motion and the angle of the sun. Because of the complexity of calculation formula, so this paper proposes to provide time by $\left(t_{0}, t_{1}, t_{2}, \cdots, t_{n}\right)$ position of the solar elevation angle $\left(x_{0}, x_{1}, x_{2}, \cdots, x_{n}\right)$ construct a simple continuous function $p(t)$ to approximate the replacement function $f_{1}(\delta(t), \omega(t))$; Similarly through the solar azimuth angle $\left(y_{0}, y_{1}, y_{2}, \cdots, y_{n}\right)$ construct a simple continuous function $k(t)$ to approximate the replacement function $f_{2}(\delta(t), \omega(t))$; The solar position calculation can be transformed into the Lagrange interpolation function to solve the problem, and it's mathematical model is as follows:

$$
\begin{aligned}
& p(t)=a_{0}+a_{1} t+a_{2} t^{2}+\cdots+a_{n} t^{n} \\
& k(t)=b_{0}+b_{1} t+b_{2} t^{2}+\cdots+b_{n} t^{n}
\end{aligned}
$$

In the equation (5): $p(t) \longrightarrow$ Solar elevation angle is calculated by interpolation;

In the equation (6): $k(t)-$ Solar azimuth angle is calculated by interpolation;

In the equation (5): $t$ - Time.

\section{Solar position calculation}

\section{Algorithm Description}

Stored in a certain date of a date by the microcontroller, the sun is located at the point of the solar setting, and the storage position information includes the solar elevation angle and the solar azimuth angle ( the solar azimuth angle at noon time is zero, the morning is negative in the afternoon).$t$ times of the solar elevation angle $p(t)$ and the solar azimuth angle $k(t)$ are obtained by interpolation calculation. Because of the large number of data points in order to avoid the errors caused by interpolation polynomial truncation error, the interpolation algorithm is used to calculate the accuracy and complexity. So we have to consider functions when the time of the whole point is odd, and the piecewise interpolation function can be used to select the quadratic interpolation functions. The interpolation function is selected as the number of time.

Linear interpolation is a line which through two points $\left(t_{0}, x_{0}\right),\left(t_{1}, x_{1}\right)$, then the quadratic expression can be derived from the straight line:

$$
q_{1}(t)=\frac{t-t_{1}}{t_{0}-t_{1}} x_{0}+\frac{t-t_{0}}{t_{1}-t_{0}} x_{1}
$$

The quadratic expression of $q_{2}(t)$, satisfy the conditions $q_{2}\left(t_{i}\right)=y_{i}, i=0,1,2$ is a parabola which through $\left(t_{0}, y_{0}\right),\left(t_{1}, y_{1}\right),\left(t_{2}, y_{2}\right)$, the equation is:

$$
q_{2}(t)=A\left(t-t_{1}\right)\left(t-t_{2}\right)+B\left(t-t_{0}\right)\left(t-t_{2}\right)+C\left(t-t_{0}\right)\left(t-t_{1}\right)
$$


This is a quadratic, as long as it satisfies the interpolation conditions, you can get the solution of the quadratic interpolation problem,

$$
\begin{aligned}
& A=\frac{y_{0}}{\left(t_{0}-t_{1}\right)\left(t_{0}-t_{2}\right)} \\
& B=\frac{y_{1}}{\left(t_{1}-t_{0}\right)\left(t_{1}-t_{2}\right)} \\
& C=\frac{y_{2}}{\left(t_{2}-t_{0}\right)\left(t_{2}-t_{1}\right)}
\end{aligned}
$$

For a given period of time in the sunshine time $t_{0}, t_{1}, t_{2}, \cdots, t_{n}$ by interval division, if $n$ is even numbers, partition interval $\left[t_{0}, t_{2}\right],\left[t_{2}, t_{4}\right],\left[t_{4}, t_{6}\right],\left[t_{6}, t_{8}\right], \cdots,\left[t_{n-2}, t_{n}\right]$; Interpolation functions can be expressed as a function of each cell:

$$
p_{i}(t)=\frac{\left(t-t_{i}\right)\left(t-t_{i+1}\right)}{\left(t_{i-1}-t_{i}\right)\left(t_{i-1}-t_{i+1}\right)} x_{i-1}+\frac{\left(t-t_{i-1}\right)\left(t-t_{i+1}\right)}{\left(t_{i}-t_{i-1}\right)\left(t_{i}-t_{i+1}\right)} x_{i}+\frac{\left(t-t_{i-1}\right)\left(t-t_{i}\right)}{\left(t_{i+1}-t_{i-1}\right)\left(t_{i+1}-t_{i}\right)} x_{i+1}, i=1,2 \cdots, n-1
$$

$p(t)$ is interpolation function of $f_{1}(\delta(t), \omega(t))$ :

$$
p(t)=p_{i}(t), t \in\left[t_{0}, t_{n}\right]
$$

If $n$ is uneven numbers, partition interval $\left[t_{0}, t_{2}\right],\left[t_{2}, t_{4}\right],\left[t_{4}, t_{6}\right],\left[t_{6}, t_{8}\right], \cdots,\left[t_{n-1}, t_{n}\right]$, Since $n$ is uneven, so the Lagrange quadratic interpolation can't be completed in the final section, so the linear interpolation is used to compensate, The linear Lagrange interpolation function is expressed as the interval $\left[t_{n-1}, t_{n}\right]$ :

$$
p_{n}(t)=\frac{t-t_{n}}{t_{n-1}-t_{n}} x_{n-1}+\frac{\left(t-t_{n-1}\right)}{\left(t_{n}-t_{n-1}\right)} x_{n}
$$

$p(t)$ is interpolation function of $f_{1}(\delta(t), \omega(t))$ :

$$
p(t)=\left\{\begin{array}{l}
p_{i}(t), t \in\left[t_{0}, t_{n-1}\right] \\
p_{n}(t), t \in\left[t_{n-1}, t_{n}\right]
\end{array}\right.
$$

The same as $f_{2}(\delta(t), \omega(t))$, The interpolation function is expressed as the quadratic interpolation function:

$$
k_{i}(t)=\frac{\left(t-t_{i}\right)\left(t-t_{i+1}\right)}{\left(t_{i-1}-t_{i}\right)\left(t_{i-1}-t_{i+1}\right)} y_{i-1}+\frac{\left(t-t_{i-1}\right)\left(t-t_{i+1}\right)}{\left(t_{i}-t_{i-1}\right)\left(t_{i}-t_{i+1}\right)} y_{i}+\frac{\left(t-t_{i-1}\right)\left(t-t_{i}\right)}{\left(t_{i+1}-t_{i-1}\right)\left(t_{i+1}-t_{i}\right)} y_{i+1}, i=1,2 \cdots, n-1
$$

Linear interpolation is expressed as:

$$
k_{n}(t)=\frac{t-t_{n}}{t_{n-1}-t_{n}} y_{n-1}+\frac{\left(t-t_{n-1}\right)}{\left(t_{n}-t_{n-1}\right)} y_{n}
$$

$k(t)$ is interpolation function of $f_{2}(\delta(t), \omega(t))$ :

$$
k(t)=\left\{\begin{array}{l}
k_{i}(t), t \in\left[t_{0}, t_{n-1}\right], \\
k_{n}(t), t \in\left[t_{n-1}, t_{n}\right] .
\end{array}\right.
$$

Based on the calculation formula of height angle and azimuth angle, we can solve the problem that the elevation and the azimuth of the traditional solar position are calculated by the interpolation. 


\section{Implementation of the algorithm}

1. Basic data set, with the solar tracking device installation position of longitude and latitude data, setting calculation date interval $\mathrm{N}$, namely the sampling period.

2. Every $\mathrm{N}$ days once the hour the solar position stored data.

3. In the process of actual operation, call a stored data, according to the type of sampling interval (12) and (16) $\mathrm{N}$ days one day the hour for the interpolation calculation, it is concluded that during the period of the hour that day the solar elevation angle and solar azimuth angle.

4. According to (15) and (18) the interpolation calculation, get any moment the solar elevation angle and solar azimuth angle.

5. According to the solar elevation angle and solar azimuth interpolation calculation results for coarse solar tracking device.

6. After rough adjustment according to the photoelectric sensor feedback signal to control the solar tracking device to realize accurate tracking.

\section{Simulation analysis}

Taking Changchun city of Jilin province in March 21, 2015 as an example, the time of sunrise is 5:40, and the sunset is 17:40. According to the interpolation principle to select the hour the solar elevation angle and solar azimuth angle data as below:

Table 1 Solar elevation angle data

\begin{tabular}{l|l|l|l|l|l|l}
\hline Time & $6: 00$ & $7: 00$ & $8: 00$ & $9: 00$ & $10: 00$ & $11: 00$ \\
\hline Solar elevation angle & 1.72 & 12.45 & 22.75 & 32.12 & 39.86 & 44.98 \\
\hline Time & $12: 00$ & $13: 00$ & $14: 00$ & $15: 00$ & $16: 00$ & $17: 00$ \\
\hline Solar azimuth angle & 46.5 & 44.05 & 38.18 & 29.9 & 20.31 & 9.85 \\
\hline
\end{tabular}

Table 2 Solar azimuth angle data

\begin{tabular}{l|l|l|l|l|l|l}
\hline Time & $6: 00$ & $7: 00$ & $8: 00$ & $9: 00$ & $10: 00$ & $11: 00$ \\
\hline Solar elevation angle & -88.85 & -78.28 & -66.85 & -53.7 & -37.87 & -18.86 \\
\hline Time & $12: 00$ & $13: 00$ & $14: 00$ & $15: 00$ & $16: 00$ & $17: 00$ \\
\hline Solar azimuth angle & 2.43 & 23.4 & 41.7 & 56.86 & 69.6 & 80.7 \\
\hline
\end{tabular}

Through the MATLAB simulation, the variation curves of the solar elevation angle are obtained by interpolation are shown in Fig.2. The variation curves of the solar azimuth angle are shown in Fig.3, and the errors are shown in Fig.4 and Fig.5 compared with the classical Spenser algorithm.

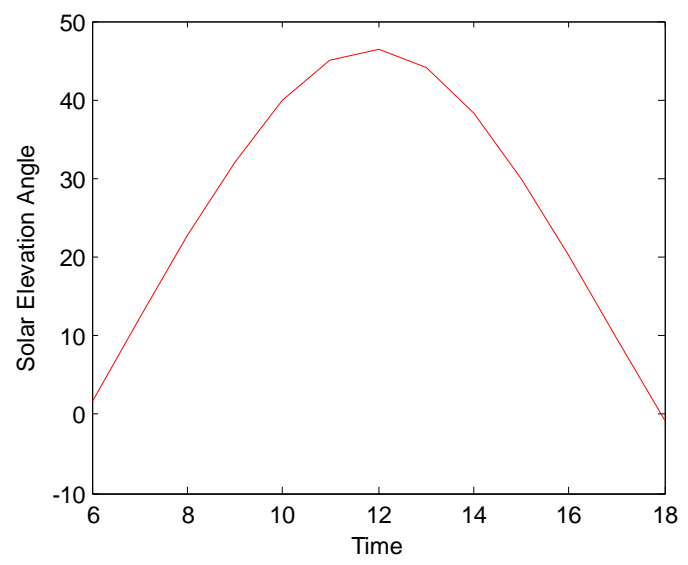

Fig. 2 Change of the solar elevation angle

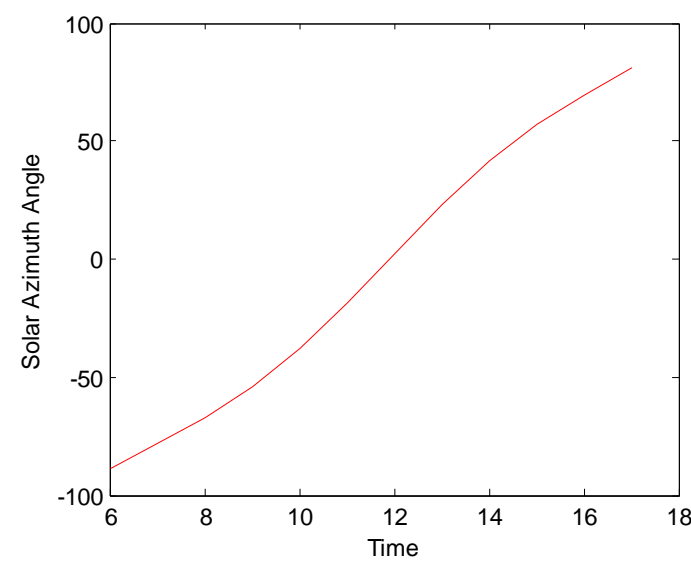

Fig. 3 Change of the solar azimuth angle 


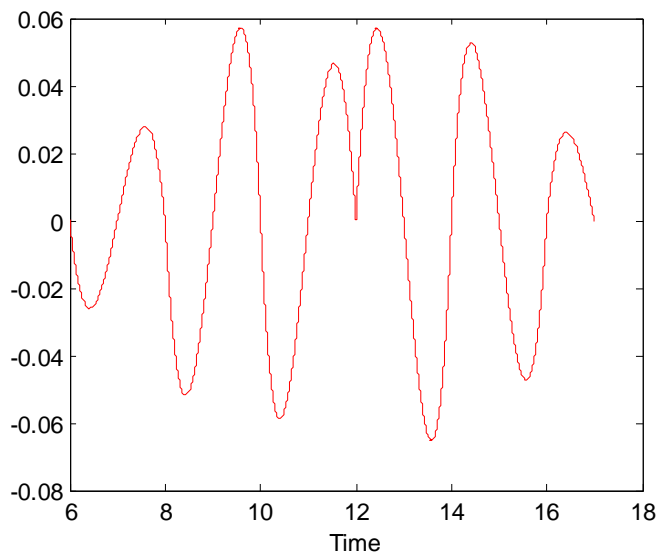

Fig.4 Error of the solar elevation angle error

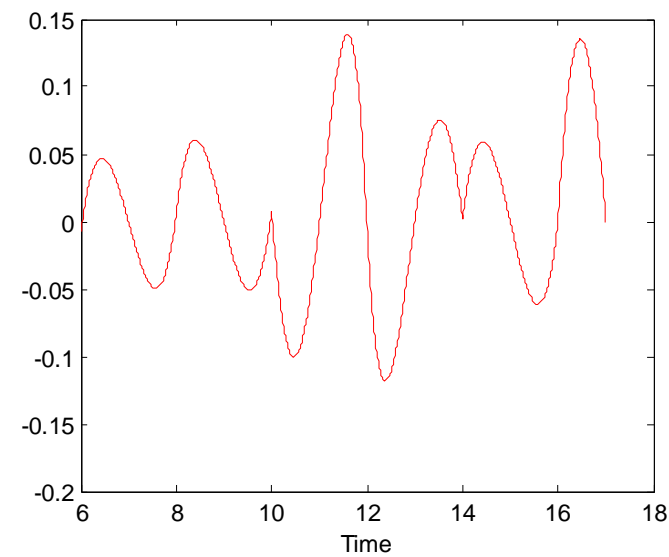

Fig.5 Error of the solar azimuth angle Table 3 The error change of different sampling period

\begin{tabular}{cccc}
\hline & $\begin{array}{c}\text { Sampling period } \\
10 \text { days }\end{array}$ & $\begin{array}{c}\text { Sampling period } \\
20 \text { days }\end{array}$ & $\begin{array}{c}\text { Sampling period } \\
30 \text { days }\end{array}$ \\
\hline $\begin{array}{c}\text { The solar elevation } \\
\text { angle error range }\end{array}$ & {$[-0.005,0.016]$} & {$[-0.55,0.1]$} & {$[-0.205,0.3]$} \\
$\begin{array}{c}\text { The solar azimuth } \\
\text { angle error range }\end{array}$ & {$[0,0.0275]$} & {$[-0.003,0.135]$} & {$[-1.03,0.126]$} \\
\hline
\end{tabular}
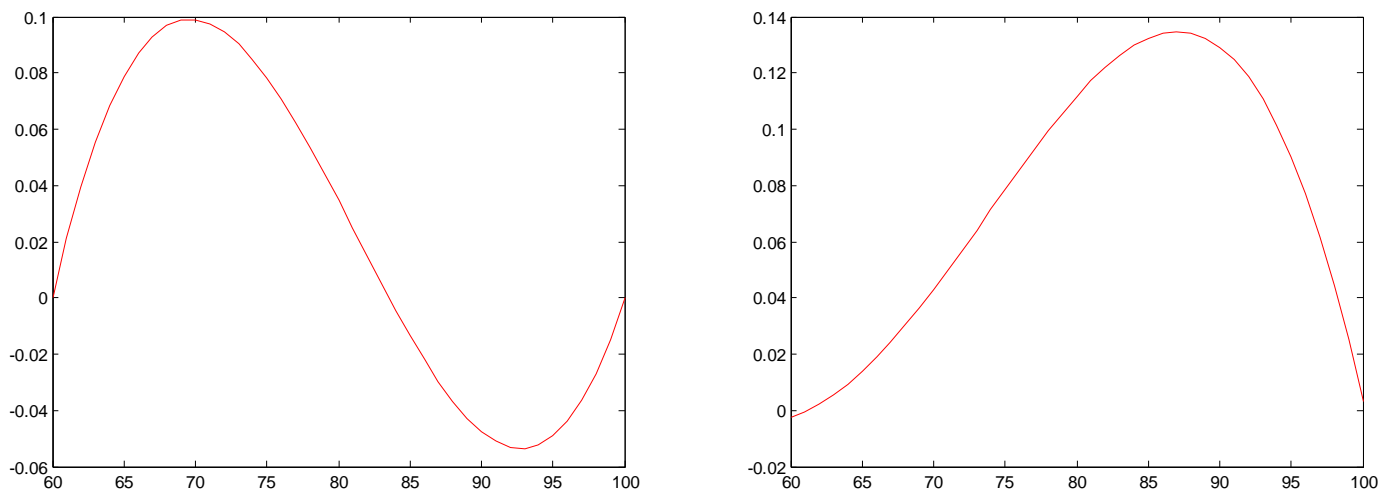

Fig.6 Error of the solar elevation angle in 20 days Fig.7 Error of the solar azimuth angle in 20 days

By the Fig. 4 and Fig. 5 we can be seen by the interpolation algorithm to calculate the solar position and the Spenser algorithm to calculate the position of solar elevation angle error range between [-0.07, $0.06]$, solar azimuth angle error in $[-0.12,0.14]$. Literature [11] has shown that the maximum error of solar elevation angle by Spenser is 0.2166 , the maximum error of solar azimuth angle is 0.3558 , by the error accumulation principle can be calculated by the interpolation algorithm of the solar elevation angle error range in [-0.2866, 0.2766], solar azimuth angle error range in [-0.4758, 0.4958].

Different sampling error variance calculated cycle solar elevation angle and Spenser by interpolation algorithm and the error of the solar azimuth angle, as shown in table 3, the overall consideration is given to the accuracy and computational complexity, it can be seen that the error range of the solar elevation angle is $[-0.55,0.1]$ in 20 days, and the error solar azimuth angle is $[-0.003$, 0.135], The error range is within the range of photoelectric sensor detection, so it can be realized that the solar position data can be calculated by the interpolation algorithm, and the sun can be tracked in order to facilitate the tracking of the solar in order to facilitate the tracking.

\section{Conclusion}

In this paper, through the simulation analysis can be obtained using Lagrange interpolation calculation method of the solar position, it can be used in the solar trajectory tracking. Under the precondition of satisfy the system requirements, it can be combined with photoelectric tracking to realize the all-round, high efficiency and high precision tracking. By using the interpolation function 
approach the solar practical operation curve, it solves the solar elevation angle and the solar azimuth angle coupling problems in calculation, it realizes the solar elevation angle and the solar azimuth angle in the tracking control system with distributed control actuator, it removes the error generated by the actuator coupling effect the precision of the system, it improves the efficiency of system calculation and simplifies the difficulty of program development.

\section{References}

[1] Y. X. Jin, Study on the dual-mode and all-weather sunlight tracking control system, Journal of Shenyang Institute of Engineering (Natural Science). 2013,9(4) 289-294.

[2] J. H. Ma, Design of a sun tracking system based on SCM Journal of Mechanical \& Electrical Engineering. 2010,27(12) 63-67.

[3] F. Pineda, Design and implementation of sun tracker prototype for solar module positioning, Photovoltaic Specialists Conference (PVSC), 2012 38th IEEE, Texas, U.S.A.2012 002905-002910.

[4] P. Xing, Design of closed-loop control system for sun tracing based on ARM, Journal of Industrial Instrumentation \& Automation. 2010(03) 24-26.

[5] C. Gay. Performance Advantages of two-axis Tracking for Large flat-plate Photovoltaic Energy Systems , IEEE Photovoltaics Specialists Conference, San Diego, CA, USA, 1982 1368-1371.

[6] X. G. Bian, An auto matic sun-tracking method in large rance. Journal of ACT A ENERGIAE SOLARIS SINICA. 2010,31(10) 1298-1302.

[7] P. Bajpai, Development and Performance Test of an Automatic Two-Axis Solar Tracker System, Energy.2002, 27 (6) 579-590.

[8] H. Z. Ping, Sun position algorithm based on T-S fuzzy model, Journal of Shanghai Maritime University. 2014,35(2) 81-84.

[9] F. Zhang, Study on contrasting model and application for simplified solar position algorithms, Journal of ACT A ENERGIAE SOLARIS SINICA. 2012,33(2) 327-332.

[10]Camacho E F, Berenguel M, Alvarado I, et al. Contral of solar power systems: a survey, Proceedings of the 9th International Symposium on Dynamics and Control of Process Systems(DYCOPS 2010), Belgium, 2010 809-810

[11]C. X. Du, A high accuracy algorithm for the calculation of solar position, Energy Engineering. 2010, (2) 41-44. 